\title{
Index
}

Page numbers in italics indicate figures

Absenteeism 347-9

ACAS

Arbitration Scheme 379

Code of Practice on Discipline and Grievance Procedures 366, 373, 374, 377

health, work and wellbeing checklist 322

Accident Book 413

Accidents

investigation 415

reporting 413-14

Accommodation

for patients see Cages, Kennels

for staff

gas safety 418

lighting 22

for live-in staff 277-8

for overnight duty staff $276-7$

ventilation 20

Accounts see Finance

Administration

activities 262-3

correspondence 272-5

filing 270-1

handling payments $268-70$

disaster recovery 280

offices 41

design 263-5

equipment 265-8

lighting 22

requirements 42

ventilation 20

staff roles 316, 356

Admission procedures

client handouts $114-15$

for infectious cases 111

questionnaires 114, 123

for surgical cases 115, 116

ADSL (asymmetric digital subscriber line) 66-7

Advertising

services and products 472

for staff 325,361

Advisory, Conciliation and Arbitration Service see ACAS

Air break receiver 26

Air-source heat pumps 18
Alarms

carbon monoxide 418

fire 30

intruder 28

Ambulances 278-9

AMTRA 386

Anaesthesia

agents 140

health and safety 161-2

endotracheal tubes 141-2

machines/breathing systems 129, 130, 139-41

SOP 167

monitoring 143-5

ECG 199

patient warming 145-6

record-keeping 160-1, 168-9

staff roles 157

ventilators 141

Analysers

for biochemistry/endocrinology 235-6

costs 234

for haematology 236

maintenance/monitoring 240

purchasing considerations 234-5

quality assessment/control 240-1

reliability 234

veterinary validation 234

Angry clients 293

Animal abuse 391

Animal breeding 390

Animal handling 93, 113

Animal welfare 388-91

Animal Welfare Act 2006387

Ansoff marketing matrix 471

Antimicrobial drug use policy 258-9

Antiseptic solutions for skin preparation 135

Appointed Persons 399, 421

Appointment systems 79

Appraisals see Staff management

Archaeology of prospective location 5

Area Safety Officer 398, 420

Arkansas stone 181

Arthroscopy 188

Asbestos surveys/regulations 9-10

Associate Veterinary Surgeon, example job description 353

Auriscopes 90

cleaning 94 
Autoclaves 151-2 hazards 162

Automatic call answering 68

BACS payments 269

Bacteriology 238

Bain breathing system 141

Balance sheet 444-6, 459

Bank workers 369

Banking risk assessment 284 timing 451

Barcode readers, and stock management 255

Barnsley unit 26

Barrier nursing 108, 111, 112

Batch numbers (medicines) 248, 255

Baths/bathing 108, 117-18

Battery disposal 53

Bedding 102 laundering 111

Behaviour policy 324

Benches, laboratory 231-2

Benchmarking 504-5

Bicycle parking 33

Bills see Invoices

Binocular loupe 133

Bins for cytotoxic waste 52 for dog waste 33 hands-free access 47 for offensive waste 53 for salt/grit 51 for sharps 95 (see also Waste management)

Biopsy endoscopic forceps 187 ultrasound-guided 206

Biosecurity in consulting rooms $93-4$

in dentistry 180

in surgical areas disinfection 151

hand/personal hygiene 150-1 protocol 170-1 and traffic flow 131 (see also Sterilization) and ventilation 20 in ward areas 109-12

Bird's triangle 414

Birds, restraint of 93

Blogging 475

Blood coagulation analysers 237 pressure monitors $144-5$ sampling tubes 233

Bluetooth terminals for payment handling 65

Body language 285-6

Bone cement 162

Bonus schemes for staff 346, 347

Bowie Dick tape 153, 154

Bradford Factor 348

Brand ambassadors 494

Branding 466-8

Breathing systems/circuits see Anaesthesia

Bribery Act 2010388

Broadband 60

Bronchoscope 187
Browne's tubes 153

'Brownfield' sites 5

Budgetting 446-7, 457-9

Building Regulations 9

Building Warrant 9

Bullying 320, 321

Business cards 274

Business plan elements 431-3 implementation 433-4 and leadership 307-8

BSAVA 384

BVA 384

BVNA 385

BVU 385

\section{Cabling 58-9}

Cadavers, storage and disposal 41, 52

Cages 37-8, 99 cat-friendly 101 cleaning 110-11 (see also Kennels)

Callipers 211-12

Cameras

for endoscopy 188-9 practice policy 282 (see also CCTV)

Capnographs 143, 144

Car parks

barriers 29

bay marking 8, 33, 35

lighting 33-4 maintenance 51-2 signage 33

Carbon dioxide for endoscopy 189

Carbon monoxide detector/alarm 418

Care plans 115-16, 124

Cars see Vehicles

Cascade see Medicines

Cash

flow 438,457

payments 269, 451 security 72

Cassettes (radiographic) 212-13

Casual workers 369

Cat catchers 93

Cat-friendly practice 508

CCTV

for building security $27,28,276-7$

employee surveillance 372

for patient monitoring 118

Ceilings 44-5

Central heating 18

Centrifuges 236

Ceramic tiling on walls 44

Certification by vets 393-4

Cesspools 4

Change

curve 310

management leadership of 309-10

in reception/waiting area 83-4 and stress 311

Change of Use Consent 7

Changing rooms 130

Charge coupled device (CCD) detectors 214

Cheque payments 269

Child abuse 391 
Circle breathing system 141

Circulating nurse role 157

Cleaners 50-1, 356

Cleaning

of air grilles and fan units 21

of endoscopes 191-2, 195-6

equipment for 40,50

storage 107

of flooring $74,75,164$

of isolation unit 113

schedule 109

staff responsible 50

standards/audit 49-50, 55

of ultrasonography equipment 206, 207

of ward areas 110-11

Client(s)

access to clinical records 392

communicating with

breaking bad news 294

the consultation 286-90

dealing with emotions 293

fees and estimates 292-3

informed consent 291-2

about inpatients 120-1

in reception/waiting area $76-7$

and service quality $480-1,487$

complaints 294-5, 488

compliance 291, 486-7

feedback 295-6

information for

on admission 114-15

in consulting rooms 91

at discharge 121

interaction points 489

meetings with 296

perceptions, and branding 467

privacy 36,85

retention strategies $469-70$

risk assessment 405-6

'touch points' 65

transfer 392

withdrawal of service 394

(see also Customer(s))

Clinical audit 508-10, 513-14

Clinical governance

benchmarking 504

client experience 508

clinical audit 508-10, 513-14

clinical effectiveness $506-8$

CPD 502-3

critical incident review 505, 515-20

evidence-based medicine 501-2

guidelines $506-7$

and information systems $507-8$

patient experience 508

peer review 504

practice policy 512

protocols 505

and RCVS Practice Standards 501

referrals 505-6

reflective practice $503-4$

in surgical areas 159-60, 173, 515-20

Clinical guidelines 506-7

Clinical partner, example job description 351

Clinical protocols 505

Clippers

cat-friendly 101

cleaning/maintenance 134-5, 166 in consulting rooms 89

wall-mounted 98, 129, 134

Clogs 137-8

Closed-circuit television see CCTV

Cloud computing 58

Coaching 311-13, 342

(see also Training)

Coaxial Lack breathing system 141

Cold rooms, for cadavers 41

Collars 100, 113, 115

Collective agreements 367

Comment cards 295-6

Commodity marketing 465-6

Communication

with clients

and clinical governance 508

complaint handling 294-5, 488

in consultations 286-91, 491

dealing with emotions 293-4, 491

meetings 296

obtaining feedback 295-6, 495-7, 500

protocols $77-8$

principles 76

and service quality 480,487

by telephone 490-1, 499

test results 242

with colleagues 296-7

and clinical governance 504-5, 506, 507

handling complaints 301

meetings 298-9, 506

obtaining feedback 299-300, 497, 500

principles 285-6

referrals 303

sharing information 300-1

with suppliers 302

and teamwork 333

training 297-9

(see also Correspondence, Marketing)

Community interactions 468, 489-90, 498-9

Compensation

for discrimination 363

for unfair dismissal 375,378

Competent Person (CP) 398-9, 421

Competition strategies $429-30$

Complaints

by clients $294-5,324,488$

by colleagues 301

(see also Grievance)

Compliance 291, 486-7

Compliment slips 274

Compressed air 24, 150

Computed radiography 214

Computer systems/networks 58-60

in consulting rooms 92-3

and digital radiography 214-15, 222

in office areas 265, 266-7

practice policy 282

security 271

(see also Practice Management System,

Workstations)

Concordance 291

Confidentiality 272

agreement example 324

Consent (client)

informed consent 291-2

for 'off-licence' drug use 249, 261

(see also Planning Consent)

Construction contracts 11 
Construction (Design and Management) Regulations 200710

Constructive dismissal 379

Consultation (clinical) and customer experience 491 process 287-90

Consulting rooms animal handling 93 biosecurity 93-4 cleaning audit 55 client information 91-2 computers 92-3 design $36,85-7$ equipment 89-91 flooring 87 hand-washing facilities 87 lighting 21, 22, 86-7 seating 88,89 storage 88 tables $85,87-8$ ventilation 20,86 waste disposal 94-5

Consumables, laboratory 233

Continuity of care 114, 391

Contracts of employment car use 283 legal aspects $366-8$

Contrast media 212

Control of Substances Hazardous to Health Regulations $2002(\mathrm{COSHH})$ and anaesthetic gases 161-2 and medicines 259 risk assessment 404-5 and zoonoses 112

Controlled areas see Radiography

Controlled Drugs see Medicines

Cooling systems 21

Corporation tax 439

Correspondence administration 262, 273-5

Corridors 37

$\mathrm{COSHH}$ see Control of Substances Hazardous to Health Regulations 2002

Cost of sales 435

Cost-led strategy 429, 430

Cost-plus pricing 435, 437

Counselling staff 342

Coving for flooring $42-3$

CPD see Training

Crash trolley 138

Credit card payments 66, 269

Credit terms/control 449-51

Critical incident review 505, 515-20

Crush cages 93

Customer(s)

choice/selection 493, 494

expectations $32-3,479-80$

experience assessment $495-7$ and building business 498-9 and clinical governance 508 components 480 during the consult 491 in emergencies 491 first impressions 490 information/merchandising 80-83, 492 in nurse clinics 492 process $492-4$ seeking excellence 498 telephone calls 490-1, 499

waiting times $78-9$

perceptions $32,479,489-90$

and practice organization 319

recommendation 493-4

satisfaction 481-2

service 324

disaster recovery 280

practice policy 324

the service - profit chain $478-9$ training 479

(see also Client(s))

Cytotoxic/cytostatic drugs

handling precautions 259-60

waste management 52, 56, 259, 260

Darkroom 213

Data

disposal 272

loggers, temperature monitoring 253 security $64,271-2$

Data Protection Act 1998270

and accident book 413

and client access to records 392

and consultations 92

in recruitment 363

Debit card payments 66, 269

Debt control/recovery 394, 448-51

Decision Notice (Planning) 8-9

Decontamination of site 5

Dentistry

biosecurity 180

charts 181, 183-4

equipment

handpieces 179

machines 178-9

maintenance 181

scalers 179

homecare 182

patient safety 180

radiography 177 safety 180

room/area 176-7

lighting 22

ventilation 20

training 181

Depreciation 441-2, 444

Dermatitis, avoidance 110

Design and Access Statement (DAS) 7-8

Diathermy see Electrosurgery

DICOM

and radiography $215,221-2$

and ultrasonography 203, 205

Differentiation

business strategy 429, 430

marketing strategy 465, 469

Digital radiography 214-16

Disabled access and building design 35 car park bays $8,33,35$ ramp 35 to front desk 36, 72

Discharge procedures 121 from isolation unit 112-13

Disciplinary action/procedure 349 and employment legislation 373-4 of RCVS 383-4

Discrimination in employment 372 
Discrimination continued

practice policy 320, 321

in recruitment 362-4

and unfair dismissal 378

Disinfectant use 151

Dismissal

constructive 379

and contract conditions 368

fair 377

notice 375-6

redundancy $376-7$

unfair 377-9

wrongful 379

Dispensary

cleaning audit 55

design 251-4

health and safety 259-60

legislation/regulations

Controlled Drugs 249-51

labelling 247-8

premises 246-7

prescribing cascade and consent 248-9

record-keeping 248, 251

SOPs 258-9, 261

stock control 254-6

training 258

waste management 260

(see also Medicines)

Distilled water deionizer 235

Diversification 430

Document scanners 268

Dog catchers 93

Dog waste bins 33, 100

Doorframes 45

Doormats 43

Doors

angled 39

automatic 75

fire 28,30

front entrance $34-5$

furniture 45-6

cleaning 50

hinges 45

locks/keys 27, 46

materials 45

sliding 86

strengthening 27-8

in ward areas 97

Doppler blood pressure monitor 145

Dosimeters for radiation 220

Drainage 4-5

Drapes 149

Drinking fountains for cats 101

Driving for work, practice policy 283

Drugs see Medicines

Duodenoscope 187

ECG see Electrocardiography

Electrical appliances, health and safety 417-18

Electrical waste, disposal 53

Electricity

self-generation $14-15$

site supply 5

sockets 14, 15, 38, 58, 92, 97

Electrocardiography

equipment

features 197-8 recording methods 198-9

selection 200-1

Electrochemical analysers 234, 235

Electrodes for ECG 197, 198

Electrosurgery

equipment $147-8$

hazards 162

E-mail

in marketing 477

messages 273

practice policy 273, 282

Emergency appointments 79, 491

Emergency equipment

in consulting rooms 91

in the surgical suite 138

Emergency lighting 23

Emotional intelligence/quotient 306-7

Emotions, dealing with 293-4, 491

Empathy 286, 287, 293, 294, 491

Employees

'life cycle' 361

statutory rights $370-3$

types 368-9

(see also Employment law, Staff)

Employers' liability insurance 394

Employment agencies, regulation 363

Employment law

contracts $366-8$

terms and conditions 380-1

discipline and grievances $373-5$

dismissal

constructive 379

fair 377

notice $375-6$

unfair 377-9

wrongful 379

employment status $368-70$

recruitment 361-6

redundancy $376-7$

resignation 375

statutory rights $370-3$

Empowerment of staff 331, 488

Endoscopy

equipment

cameras 188-9

endoscopes 186-8

maintenance/cleaning 191-2, 195-6

new versus second-hand 190

sterilization 190

storage 191-2

tables 189

trials 190

warranties 190

pricing 192

room set-up 185, 190-1

training 192-3

Endotracheal tubes 141-2

Energy efficiency, buildings 9, 13

Environmental impact 396

(see also Waste management)

EQ (emotional quotient) 306-7

Equal opportunities policy 320

Equality Act 2010 320, 362, 364

Estimates 293

Ethical standards 395-6

Ethylene oxide sterilizers 153, 162

Euthanasia

RCVS Supporting Guidance 389

of strays 390 
Evidence-based veterinary medicine (EBVM) 501-2

Exercise areas for dogs 41, 100-1

Exotic pet housing 101, 102

Extinguishers (fire) 30-1

Eye contact in communication 286 washing 416-17

\section{Facebook 476}

Facemasks 137 for laser surgery 163

Faecal waste bins 33, 100

Fault reporting 49

Fax machines/servers 69, 268 messages 272

Feedback from clients 295-6, 495-7 from colleagues 300, 497 and coaching 311-13

Feed-in tariffs (FITs) 15

Feeding chart 127

Fees communicating to clients 292 setting 394, 452-3 (see also Pricing)

FeLV test kits 238

Filing 270-1

Finance accountants 455-6 accounts 447 administration 262 balance sheet 444-6, 459 banking 451 budgets 446-7 and business planning 433 cash flow 438, 457 corporation tax 439 cost-benefit analysis 454-5 costs 453-4

debt control 448-51

depreciation 441-2, 444

fee/price setting $452-3$

fixed assets 442-3

income tax 439

mark-up 436-7

NIC 439

overheads 436, 438, 447

PAYE 439, 452

payroll 451-2

profit

and customer service $478-9$ gross $436-8$ and loss account 441-4, 458 net 438

profitability $452-4$

of laboratory tests 229, 230

quotes and estimates 453

reconciliation 451

software 441,451

terminology 435-6

terms and conditions $460-1$ and trading structures $439-41$ turnover 436

VAT 439,451

working capital management 448

Fire Officer 30, 398, 420
Fire safety

alarms 30

doors 28

escape 29

extinguishers $30-1$

and night staff 119

regulations 29

risk assessment 29

signage 48-9

in the surgical suite 164

testing and drills 31

First Aid

box 416

checklist 423

eye washing 416-17

personnel 399, 421

FIV test kits 238

Five Freedoms 99

Fixed assets register $442-3$

Fixed-term Employees (Prevention of Less Favourable Treatment) Regulations 2002369

Flat panel detectors 214

Floor boxes 265

Floor plans

consulting room 36

prep area 39

wards 38

Flooring

in consulting rooms 87

materials $42-3$

in reception/waiting area 74

in wards/kennels 96, 97, 99-100

Fluid therapy equipment 105-6

Follow-up after discharge 121

Food preparation area 98, 107

Form F10 10

Form P45 375

FPS payments 269

Franking 272

Freezers for cadavers 41

Front desk 35-6, 71-3

Front doors $34-5$

'Gardening leave' 375

Gardens 33

Gas (mains) 4, 13-14

appliance safety 418

regulations 12

site supply 5

(see also LPG and Medical gases)

Gas Safe Register 418

Gas Safety (Installation and Use) Regulations 1988418

Gifts, practice policy 388

Gloves 89, 110, 137

Glucometers 237

Goggles for laser surgery 163

Goodwill value 444-5

Gowns 149

GPRS terminals for payment handling 65

Grids (radiographic) 213, 214

Grief, dealing with 294

Grievance 349 practice policy 301 procedure 301, 374-5

Grooming 107-8, 117

Gross misconduct 374

Ground-source heat pumps 18

Grounds maintenance 51-2

Guilt, dealing with 293 
Haematology analysers 236

Halogen lighting

external 34

in operating theatres 132

Halothane 140

Handshaking 286

Hand-washing

facilities 36, 37, 48, 87, 94, 109-10, 134

technique 94,110

WHO guidelines 109

Harassment 320, 321, 362

Harnesses 113

Hazardous substances

in the laboratory 233

medicines 259-60

risk assessment 404-5

in the surgical suite 161-2

Hazardous waste 41, 52-3, 56, 57

Head-mounted light sources 132-3

Health and safety

accident reports/investigation 413-15

administration 263

and building construction 10

of electrical appliances $417-18$

employers' statutory duties 371

First Aid facilities 416-17

of gas appliances 418

and handling medicines 259-60

in laboratories 239

of lone workers 409-11

of mains gas 14

of mains water 17

Management Arrangements 397-8, 420-1

manual handling 113-14

and night staff 119

in office areas 264

personnel 398-9

Policy Statement 397, 419

poster 412

in practice manual 324

premises checklist $422-3$

in radiography $208,209,212,216-20,224,226-7$ risk

assessment 399-406, 408, 409, 410

control 406-7, 411

signage 48-9

staff consultation $334-5,411-12$

in the surgical suite $161-4$

training 412-13

(see also specific Acts and Regulations)

Health and Safety Advisor (HSA) 398

Health and Safety at Work etc. Act 1974 (HASAWA) 397, 419

Health and Safety Coordinator (HSC) 398, 421

Health and Safety (First Aid) Regulations 1981 399, 416

Health and Safety Information for Employees

(Amendment) Regulations 2009412

Heated bed 146

Heating 18-19

in wards 97

Holidays 348

employee rights 371

Holter ECG monitors 200

Hospital record sheets 117, 125-8, 121

Hot desking 263-4

Human resources administration 263

Hydrogen peroxide - gas plasma sterilizers 153

Hydrotherapy 108, 122

Hygiene see Biosecurity, Cleaning, Hand-washing
Identification

of patients 115

of staff $73,75,288$

Image-intensifying screens 212

Immigration, Asylum and Nationality Act 2006 364-5

Income tax 439

and staff accommodation 278

Incubators

for oxygen therapy 103, 104

for warming fluids 105

Induction loop technology 35

Induction process/training

appraisal 346

for existing staff $337-8$

for new staff $335-7$

for reception area staff $77-8$

Infectious cases 111-12

(see also Biosecurity)

Infectious waste 52, 56

Informed consent see Consent

Infra-red heaters 19

Infra-red sensors 29

Infusion pumps 105, 106, 145

Insurance

employers' liability 394

pet $395,451-2$

practice/vehicles 279-80

professional indemnity 394

public liability 394

Intensive care units 103

accommodation 103

equipment 104-6

management 120

record sheet 128

Internet

merchant services (IMSs) 65-6

practice policy on use 282

(see also Social media, Websites)

Interviews

exit 350

in recruitment, 326-8, 363-4

return-to-work 349

Intruder alarms 28-9

Investors in People 504

Invoices

breakdown 301

requirements 274

timing 449

Ionising Radiations Regulations 1999 216, 218, 219, 224, 225, 226

ISDN (integrated services digital network) 66-7

Isoflurane 140

Isolation areas/wards

design and equipment 106-7

heating 19

lighting 22

management 111-13

ventilation 20, 106

IT

computer platform/operating systems 58

disaster recovery 280

electronic payments $65-6$

and marketing 64-5

networks 58-60

policies 64

practice management system

installation 64

planning 60-2

selection $62-7$

training 64 
Jackson Rees-modified breathing system 141

Job application forms

example 357-9

legal aspects 363

Job descriptions 320

examples 351-6

in recruitment 361,325

Job offers, legal aspects 366

Kennel cough, practice policy 111

Kennels 96, 99-100

cleaning 110-11

Ketamine, record-keeping 251

Key opinion leaders 493, 498-9

Key performance indicators 431

Kneeling pads 102, 103

\section{Labels/labelling}

of medicines 247-8, 254, 257

printers 252

of radiographs 211

of sterile packs 155

in wards 117

(see also Signage)

Laboratories

benches 231-2

design 40, 231, 232

equipment 228

analysers 232, 233-6, 240-1

centrifuges 236

consumables 233

glucometers 236, 237

infectious diseases test kits $237-8$

for microbiology 238

microscopes 236-7, 243

refractometers 236, 237, 240

health and safety 239

in house versus external 228-31

lighting 22

post-mortem examinations 238

profitability 229, 230

record-keeping 241-2

results reporting 242, 245

sample submission

administration 272-3

records 241

SOPs 240, 243

sinks 232

SOPs 239-40, 243-4

staffing 238-9

storage 232-3

ventilation 20

waste management 239

Land use classification $6-7$

Lasers 148

safety considerations 163-4

Laundry facilities 40, 107

Lavatory see Toilets

'Law of the lid' 307

Lead aprons 212

Leadership

and change 309-11

feedback and coaching 311-13

leaders versus managers 305

qualities 306-7

role 307-9

and teamwork 333

time management 313-14
Leads 113

Leaflet racks 91

Leaflets see Marketing

Leave

employee rights 371,373

practice policy 348

LED lighting 22-3

Legionella 17

Legislation/regulations see specific Acts etc.

Lift for patients 136

Light beam diaphragm 210

Lightboxes 213-14

Lighting

in car parks $33-4$

in consulting rooms 86-7

dimmable 21

emergency 23

in examination areas 21

halogen 34

levels 21

low-energy 33

and monitor viewing 215

in office areas 264

in reception/waiting areas 22

risk assessment 408

in surgical areas 21, 129, 132-3

in wards 97

Limb bin 54

Linkedln 476

Linoleum 43

Liquid nitrogen 150

Liquid propane gas (LPG) 14

List building 476-7

Local Plan 4, 6

Local Planning Authority (LPA) 6, 7 consultations 8

Local Rules for radiography $219,226-7$

Location

archaeology 5

client perception 6

drainage 4,5

finding a site 2-3

ground conditions 4

previous use/contamination 5

topography 4

usable size 3-4

utilities 5

Lockers 130

Locks 27, 44

Logos 467

Lone workers 409-11

LPG see Liquid propane gas

Magill breathing system 141

Magill endotracheal tube 142

Management of Health and Safety at Work Regulations 1999 (MHSWR) 397, 399, 420-1

Managers, versus leaders 305 (see also Practice manager, Staff management)

Managing partner, example job description 352

Manual handling 113-14, 163, 399

Marketing

4 Ps 462-3

$7 \mathrm{~S}$ model $464-5$

administration 262

advertising 472

branding 463-8

checklist 477 
Marketing continued

commodity 465-6

differentiation 465

e-mail 477

and IT 64-5

list building $476-7$

literature 473

in reception/waiting area 80

offerings $470-1$

orientation 463

public relations 472-3, 498-9

'recommend a friend' 499

for retention 469-70

sales promotions 471

segmentation 468-9

social media 473-4

strategy 463

targeted 469-70

television in reception/waiting area 80-1

video 476

website $473-5,490$

Maternity leave 349, 371

Matrix organizational structure 318

Mats

in consulting rooms 88

in kennels 99

in office areas 264

in waiting areas 74-5

McKinsey Model 464-5

Mechanical ventilators 141

Medical gases

cylinders 23, 24, 150

hazards 163

piped 24-5

scavenging 25-7, 146

(see also specific gases)

Medicines

adverse reactions 249

audit 248

classification 247

consent 248, 249, 261

Controlled Drugs

cabinet 250

disposal 53, 251, 260

Register 250-1

Schedules 250

dispensing

authorization 256-7

containers/packaging 257-8

labelling 247-8, 254, 257, 261

SOPs 258-9, 261

training 258

display regulations 82

disposal 52, 53, 56, 251, 260

hazardous substances 259-60

importation 249

prescribing 247

cascade 248-49, 261

repeat 257

record-keeping 246, 248, 251

SPCs 249

stock control 254-6

storage 98, 252-4

supplier selection 255-6

Meeting rooms 275

Meetings

with clients 296

with colleagues 298-9, 300, 334, 500, 506
Mentoring 342

(see also Coaching, Training)

Merchandising in reception/waiting area 81-3

Merchant services 65

Microbiology 238

Microchipping strays 390

Microscopes 236-7, 239, 243

Mini-Lack breathing system 140, 141

Minor procedures area 107

Misconduct 374

Mission statement $330-1$

Mobile phone policy 276

Monitoring machines see Anaesthesia and specific monitors

Mortuaries 41

Motivating staff $331-2$

Moving annual turnover (MAT) 301

Muzzles 93

Mystery shoppers 490, 495

National Insurance Contributions 439

Natural ventilation 19

Negotiation skills 302

Net promoter score (NPS) 497

Networks see Computer systems/networks

Newsletters 80

Night workers 372-3

(see also Lone workers)

Nitrous oxide 24, 25, 150

Noise, in offices 264

Non-hazardous waste 53-4, 56, 57

Notice of dismissal 375-6

Noticeboards, in reception/waiting area 75,80

Nurse coordinators 157-8

Nurses see Veterinary nurses

Occupational Safety and Health Consultants Register 399

Oesophageal stethoscopes 143

Offensive waste 41,53

Offices see Administration

Official veterinarians 394

Oil boilers/tanks 14

Operating theatre

cleaning 151, 170-1

design/layout 39-40, 131

equipment 146-9

finishes 133-4

health and safety $161-3$

lighting 21, 22, 23, 132-2

set-up sheet $174-5$

ventilation 20,133

(see also Preparation area, Surgery)

Ophthalmoscopes 90

Oscillometric blood pressure monitor 145

Outline Consent (Planning) 7

Out-of-hours cover 391

Overnight care 118-19

Oxygen

cage 104

concentrators 23-4

cylinders and storage 23, 150

emergency cylinder 91

generator 104

piped 25

therapy $103,104-5$

in ward areas 97, 104 
P2P payments 66

PABX 67-8

PACS (picture archiving and communication system) 215, 221

Paging systems 69

Parking see Car parks

Partnership 1, 440

Part-time Workers (Prevention of Less Favourable Treatment) Regulations 2000 368-9

Passive infra-red sensors see PIR sensors

Pass-through cabinets 40, 134

Patch panel 59

Paternity leave 371

PAYE 439, 452

Payment, employee rights 371,452

Payments received, handling $65-6,268-70$

Payroll 439, 451-2

PCV, SOP 244

PDQ machines 65

Performance-related pay 346-7

Perioperative fatality, review form 515-20

Personal protective equipment (PPE)

for dentistry 180

for handling cytotoxic/cytostatic drugs 259

in isolation units 106, 107

for post-mortem examinations 238

for radiography 212,226

in surgical areas 136, 137, 163-4

(see also specific items)

Personal skills profile 325

Pet insurance 395 credit control 451-2

Petty cash 269

Pharmaceuticals see Medicines

Pharyngeal packs 180

Photocopiers 267-8

Photographic chemicals, disposal 53

Photographs, practice policy 282

Photovoltaic panels 15

Physiotherapy 108, 122

PIR sensors

external 34

internal 29

Planning Consent application process 7-8

Decision Notice 8-9

land use classification 6-7

Local Plan 6

Planning Officer 4, 8

Plant rooms $12-13$ heating 19

Plants, in offices 264

PMS see Practice management system

Polymethylmethacrylate (PMME) 162

Porter's five forces 428-9

Positioning aids for endoscopy 189 for radiography 211 for surgery 146-7

Positive action/discrimination 362-3

Postage, payment options 272

Postcodes and client scatter 3

Posters

hand-washing 110

health and safety law 412

in reception/waiting area 80

Post-mortem examinations 238

Power see Electricity

PPE see Personal protective equipment
Practice development

building regulations/legislation 9-10

construction phase 10-11

expansion versus relocation 1-2

planning process 6-9

professional support 2-3, 11

site review/selection 2-6

Practice management system (PMS)

access via mobile devices 60

and clinical audit 509

financial systems 441,451

functionality requirements $61-2,65$

hardware 62

installation 64

and laboratory results 241-2

planning 60-1

selection 62-4

support 62

training 64

Practice manager

example job description 354

role 309,316

Practice manual 322-4, 507

Practice owner role 315

Practice policies 301, 322, 348

examples:

anti-discrimination 321

behaviour 324

bullying and harassment 321

clinical governance 512

computer/camera/Internet/e-mail use 282

CPD 323

driving for work 283

equal opportunities 320

gifts 388

kennel cough 111

personal hygiene and appearance 323

Practice Safety Officer 398, 420

Practice Standards Scheme see RCVS

Practice values 330

Pregnancy, and radiography 220, 227

(see also Maternity leave)

Preparation area/room

checklist 159

design/layout 38-9, 129-30

equipment

for emergencies 138

for patient monitoring 143-5

for patient transport 135-6

for patient warming 145-6

for skin preparation 134-5

surgical attire/PPE 136-8

tables 129, 130, 134

lighting 22, 129

ventilation 20

workstations 130

(see also Anaesthesia, Medical gases, Sterilization)

Prescribing see Medicines

Prescription notice 247

Press releases 473

Pressure test, buildings 9

Pricing

cost-plus 437

elasticity 463

endoscopy 192

laboratory tests 229

medicines 452-3

professional fees $452-3$

Printers 267

for labels 252 
Privacy

and correspondence 273

in office areas 264

in staff accommodation 277, 278

Procedure review form 173

Process mapping 483-5

Processors (radiographic) 213, 220

Product life cycle 470-1

Professional conduct see RCVS Code of Professional Conduct

Professional indemnity insurance 394

Profit see Finance

Promotion see Marketing

'Prophy' paste 180

Protocols (written)

for client interaction $77-8$

and clinical governance 505

(see also Practice policies, Standard operating procedures)

Public address system 68

Public liability insurance 394

Public relations $472-3$

Pulse oximeters 143-4, 145

Quality

assessment/control in laboratories 228-9, 240-1 of service $481-3,487$

Questionnaires

of customer feedback 495-7

pre-admission 114, 123

Rabbit housing 101, 102

Radiation dosimetry 220, 226

Radiation Protection Advisor (RPA) 216, 217, 218, 225

Radiation Protection Supervisor (RPS) 217, 218-19

Radiators 18-19

Radiography

controlled areas 208-9

darkroom 213

dental 177,180

digital

changing to $215-16$

equipment $214-15,221-2$

financial considerations 216

terminology 215

equipment

callipers 211-12

contrast media 212

detectors 214

films/screens/cassettes 212-13, 221

grids 213,214

markers 212

positioning aids 211

processors 213, 220

tables 210-11

viewers 60, 90, 147, 213-14, 214-15

X-ray machines 209-10, 214

health and safety 180, 208, 209, 212

dosimetry 220, 226

Local Rules 219, 226-7

notification of work 217, 225

PPE 212, 226

pregnancy/breast-feeding 220, 227

risk assessment 216-17, 224

RPA/RPS 217-19, 225, 226

image storage/sharing 221-2

quality control $213,220-1$ record-keeping 222

room/area

cleaning audit 55

controlled areas 208-9, 226

lighting 22

training 223

waste management 223

Radiotelemetry, ECG recording 199-200

RAID servers 221

RCVS

Code of Professional Conduct 382-3

and out-of-hours cover 391

and prescribing 247

principles of practice 395

for veterinary nurses 383

disciplinary process 383-4

Practice Standards Scheme 32, 486

and clinical governance 501

and CPD record cards 342

and dispensing $247-8$

staff identification 288

written protocols required 78

regulatory role 382

Reception/waiting areas

change management $83-4$

cleaning audit 55

design $35-6,70-6$

key tasks 70

lighting 22-3

process mapping 485

ventilation 20

Receptionist

empowerment 331

example job description 355

induction/training 77-8

recruitment 328

role $70,76,316$

'Recommend a friend' schemes 499

Reconciliation of accounts 270

Record-keeping

and clinical governance 508

in the dispensary $248,250-1$

in laboratories 241-2

for radiography 222

in the surgical suite $160-1,168-9,172,173$

in training $341-2$

Recovery area

design and equipment 102-3

gas scavenging 26-7

Recruitment

advertising 325, 361-3

application form example 357-9

candidate assessment 328-9

contracts of employment $366-8$

eligibility to work in UK 364-6

interviewing 326-8, 363-4

the job offer 367

personal skills profile 325

references 325-6, 360

Recycling waste 53

Redundancy 375, 376-7

Reed bed sewage treatment 4, 5

Referrals 303, 391-2, 505-6

Reflective learning/practice 342, 503-4

Refractometers 236, 237, 240

Refrigerators

in laboratories 232

for medicines 253

in staff rest areas 275-6 
Regulations see specific regulations

Relocation, reasons and options 2

Reportable diseases 414

Reportable injuries 413-14

Reporting of Injuries, Diseases and Dangerous Occurrences Regulations 1995 (RIDDOR) 413, 414

Representative of Employee Safety (RES) 411

Resident Labour Market 365-6

Resignation 375

Rest areas for staff $20,22,41,42,275-6$ periods, entitlements 373

Retention strategies (clients) 469-70

Risk

assessment

examples 284, 224, 408, 409, 410

legislation 399-401

process 402-9

radiation $216-17,224$

fire 29

medicines 259

zoonoses 112

Role play 298

Roofspace use 41

Royal College of Veterinary Surgeons see RCVS

S survey 3

Safes 268

Safety, of patients, checklists 160, 172 (see also Health and safety, Security)

Sales and marketing plan 433

SARSS 249

SBEM 9, 13

Scalers (dental) 179

Scales

in consulting rooms 89-90

in reception/waiting area 72 in wards 107

Scanners see Document scanners

Scavenging of waste gases 25-7, 146

Schedule 3 procedures $386-7$

Schrader outlets 24, 25

SCORPIO marketing strategy model 463

Screening (consultation technique) 288

Screens

image-intensifying 212, 221

radiation protection 208

Scrub nurse, role 157

Scrub sink 134

Scrub suits $130,136-7$

Seating

in consulting rooms 88,89

in reception/waiting area 73

for ultrasonography 204

Security

alarms and sensors 28-9, 34

CCTV 27, 28, 277

doors and windows 27-8, 33-4, 46

and lone workers 411

and night staff 119

in reception/waiting area 36,72

of staff accommodation 276-8

(see also Biosecurity, Fire safety)

Self-employed persons 369-70

Septic tanks 4, 5

Severance pay 376

Sevoflurane 140

Sewage treatment $4-5$
Sharps disposal 52-3, 56, 94-5

Short-time working 371

Showers 108

Sickness, employee rights 348,371

Signage

external 33,48

internal doors 48-9, 86

in isolation units 106

safety/warning signs 48, 164, 208, 209, 260, 405, 407

Simplified Building Energy Model (SBEM) 9, 13

Sinks $47-8$

in cleaning cupboard 40

in consulting rooms 87

in laboratories 232

in surgical areas 133-4

Situational leadership model 312

Skype 68

SMART objectives, and strategic planning 431

'Smart' phones 69

Social media and marketing 64-5, 475-6

Social Security (Claims and Payments) Amendment Regulations 2011413

Solar energy 15

Sole traders 440

Solid fuel energy 14

Sonic scalers 179

SOPs see Standard operating procedures

Space heating see Heating

SPCs 249, 405

Special Import Certificate (SIC) 249

Special Treatment Certificate (STC) 249

Spectrophotometric analysers 234, 235

Splays 4

Staff management

absenteeism/sickness 347-8

behaviour policy 324

communication/consultation 296-303, 333, 334-5, 411-12

disciplinary process $349,373-4$

empowerment 331, 488

equal opportunities 320

exit interviews 350

grievances 301,349

handbook 322

job descriptions 320, 351-6

motivation 331-2

organizational structures $316-17$

performance

appraisal 343-6

and pay $346-7$

problems 347

return to work 349

roles and responsibilities 315-19

teamwork 308, 311, 313, 332-4

wellbeing 321-2, 348, 392, 406

(see also Dismissal, Employment law, Health and safety, Recruitment, Security, Training and specific practice areas and roles)

Standard operating procedures (SOPs)

for barrier nursing 111, 112

for the dispensary 257, 258, 261

for endoscopy 194-6

for inpatient care 120

in laboratories 239-40, 243-4

for surgical areas/equipment 156, 159, 166, 167, 170

for ultrasonography 207

Stand-offs for ultrasonography 204

Stationery 273-5

Statutory rights of employees $370-3$ 
STEEPLE model 425-6

Sterilization

autoclaves 151-2

chemical 152-3

dry heat 152

of endoscopes 190, 194

indicators 153-4

ionizing radiation 153

plasma 153

wrapping for 154-5

Stethoscopes 90

Stock

oesophageal 143

management 448

in dispensary 252, 254-6

value 445

Stools

for dentistry 176, 177

in operating theatres 147

for ultrasonography 204

Storage

of cadavers 41,54

of computer records 271

of endoscopes 191-2

for hazardous chemicals 164, 233

of intravenous fluids 105

of medicines 250, 252-4

office areas 262

of scrub suits 130

of sterile items 155

under-cage 38

units

in consulting rooms $88-9$

in the dispensary 252

in laboratories 232

materials/design 46-7

in prep areas 130

of waste 54

Strategic planning

cost versus differentiation leadership 429-30

implementation 431

leadership 308

models

Porter's five forces 428-9

STEEPLE 425-6

SWOT 426-7

process 424

Stray animals 390

Stress, and change 311

Stretcher

station 98

use 136

Suction machines 103, 148

Suggestion form/schemes 300, 304

Suitably Qualified Persons 246, 386

Surgery

attire $136-8,149,158$

instruments 147

cleaning 155-6

hazards 162

list management 158-9

patient admission/handover 115

team roles $157-8$

(see also Operating theatre, Preparation area/room)

Surgical site infections (SSIs) 151

Surveillance by employers 372

Surveys of customer feedback 495-7

Suspected adverse reaction surveillance scheme

(SARSS) 249
SWOT analysis

in practice development 1

in strategic planning $426-7$

Syringe drivers 102, 105, 106

Tables

in consulting rooms $85,87-8$

for dentistry 176,177

for endoscopy 189

heated 146

in prep areas 129, 130, 134

for radiography $210-11$

for surgery 146

for ultrasonography 204

Tail docking, and Animal Welfare Act 389

Taps 48, 134

Targeted marketing 469

Teams/teamwork

coaching 313

commitment 308

feedback 497-8, 500

management 332-4

selection 311

Telecommunication systems 66-9

Telemedicine 222

Telephone

ECG recording systems 199

message-taking 273

policy 276

protocol for inpatient owners 120

skills 77

and customer experience 490-1, 499

systems 67-9

disaster recovery 280

in surgical areas 134

Teleradiology 222

Television

closed-circuit see CCTV

licence 276

in reception/waiting area 80-1

Temperature probes 145

Temporary staff, legal aspects 369

Terms and conditions

of business 293, 460-1

of employment 380-1

Theatre see Operating theatre

Thermometers

for medicines storage 253

for patients 90

Time management 313-14

Tissue staining kits 237, 240

To and fro breathing system 141

Toilets

lighting 22

in reception/waiting area 73, 74

inspection checklist 50

ventilation 20

Tonometers 90, 91

Topography of prospective locations 4

Total quality management (TQM) 482-3

Touch 294

Trade Unions, employee rights 371

Trading structures 439-41

Traffic flow

in clinical areas 37

in surgical areas 131

Training

and clinical governance 502-3 


\section{Training continued}

in communication skills 297-8, 499

in customer service 479

for dentistry 181

for dispensary 258, 386

for endoscopy 192-3

and health and safety $412-13$

management of 335-42

for physiotherapy 122

in practice ethos 494

practice policy on CPD 323

for radiography 223

for reception area staff 77,83

record cards 341-2

for ultrasonography 206

(see also Coaching)

Transfer of Undertakings (Protection of Employment)

Regulations 2006 368, 369

Transporting patients 135-6, 391

Trolleys

anaesthetic 135-6, 139

for ECG equipment 199

for endoscopy equipment 185, 190

flatbed 256

for gas cylinders 163

for surgical instruments 162

for ultrasonography 202, 204

TST strips 153, 154

Tub tables 134

for dentistry 176, 177

TUPE see Transfer of Undertakings (Protection of Employment) Regulations 2006

Turnover 436

Twitter 476

UK Shortage Occupation list 365, 366

Ultrasonic cleaners for instruments 156

Ultrasonic scalers 179

Ultrasonography

biopsy 206

clipping 206

image viewing/storage 205

restraint for 205-6

room design 205

SOP 207

stand-offs 204

tables and seating 204

training 206

transducers 203-4 cleaning/storage 206

ultrasound machine 202-3

cleaning/maintenance 206, 207

Underfloor heating 19

Underwater treadmill 108

Unfair dismissal 377-9

Utilities

disaster recovery 280

future proofing 12

regulations 12

(see also Electricity, Gas, Water)

Vaccines, refrigerated dispensers 89, 92

Vacuum cleaners 135

Value (as marketing concept) 470

(see also Finance)

Value added tax (VAT) 439, 451
Vaporizers 139-40

VDU see Workstations

Vehicles

allowances 279

insurance 280

practice policies 283

Ventilation

air movement systems 19-20

and biosecurity 20

in consulting rooms 86

in isolation units 106

natural 19

in office areas 264

in operating theatres 133

in wards 97

Veterinary Defence Society (VDS) 385

Veterinary medicinal products see Medicines

Veterinary Medicines Directorate (VMD) 385

Veterinary Medicines Regulations 246, 247, 258, 385

Veterinary nurses

badges/cards 387

clinics 492

Code of Professional Conduct 383

delegation to 387

disciplinary process 384

example job descriptions 353, 355

List and Register 383

recruitment 328

roles and responsibilities 316

surgical 157-8

team management 114

Veterinary Practice Management Association (VPMA) 385

Veterinary Surgeons Act 1966 386-7

Victimization 362

Video

camera for endoscopy 189

marketing 476

use in training 298

Vision splays 4

Visiting

isolation cases 112

policy 120-1

Voice over Internet Protocol (VoIP) 68

Waiting

areas see Reception/waiting areas

times 78-9

Wall finishes $43-4$

Ward rounds 114

Wards

admitting patients $114-16$

caging/kennels 37, 38

bedding 102

design/materials 99-100

for cats 101

for dogs 100-10

for rabbits/exotics 101, 102

cat-friendly 101

client communication 120-1

design 20, 22, 37-8, 96-7

discharging patients 121

equipment 97-9

health and safety 113-14

hygiene/biosecurity 109-13

inpatient care

animal handling 113

bathing 117-18 
Wards, inpatient care continued

fluid therapy 105-6

grooming 117

overnight 118-19

oxygen therapy 104-5

protocols 120

nursing team management 114, 119

support areas 107-8

(see also Intensive care unit, Isolation unit, Recovery area)

Warming aids 102, 145-6

Waste management $52-5,56-7$

in consulting rooms $94-5$

in laboratories 239

of medicines 251, 260

of radiographic chemicals 223

Water

deionizer 235

gap 16-17

health and safety 17

recycled 17-18

regulations 16

softeners 17

solar heating of 15

supply 5,16

tanks 17, 18

Webforms 476

Websites 473-4, 490
'Whistle blowing' 372, 392-3

Wildlife treatment 390-1

Wind power 15

Windows, security 28

Wireless networks 59-60

Workers, legal definitions 369

Working Time (Amendment) Regulations 2003 372, 392

Working Time Directive 276, 369, 373

Workplace (Health, Safety and Welfare) Regulations 1992422

Workstations

design/assessment 265-6

in prep areas 131

risk assessment 93

Worktops 47

Wound classification 131

Wrongful dismissal 379

WSAVA, clinical guidelines 507

X-ray machines 209-10, 214, 221

Young workers 373

Zero hours workers 369

Zoonoses, risk assessment 112 